\title{
Exercises
}

The exercises are organized in problem sets following the developments in the main text. In particular, Chapters 1-3 are well covered with exercises. Some of the problem sets have the character of small projects, and many of them have been used as part of the course work in connection with a course aimed at advanced undergraduate and beginning graduate students at the Technical University of Denmark.

\section{Problem Set 1}

Exercise 1.1. Let $\left(X, d_{X}\right)$ and $\left(Y, d_{Y}\right)$ be metric spaces.

Define $d_{X \times Y}:(X \times Y) \times(X \times Y) \rightarrow \mathbb{R}_{0}^{+}$by

$$
d_{X \times Y}\left(\left(x_{1}, y_{1}\right),\left(x_{2}, y_{2}\right)\right)=\max \left(d_{X}\left(x_{1}, x_{2}\right), d_{Y}\left(y_{1}, y_{2}\right)\right) .
$$

1) Show that $d_{X \times Y}$ is a metric on $X \times Y$.

2) Show that the projections

$$
\begin{aligned}
& p_{X}: X \times Y \rightarrow X, \quad p_{X}(x, y)=x \\
& p_{Y}: X \times Y \rightarrow Y, \quad p_{Y}(x, y)=y
\end{aligned}
$$

are continuous mappings.

Exercise 1.2. Let $(S, d)$ be a metric space. For every pair of points $x, y \in S$, we set

$$
\tilde{d}(x, y)=\frac{d(x, y)}{1+d(x, y)} .
$$

Show that $\tilde{d}$ is a metric on $S$ with the property $0 \leq \tilde{d}(x, y)<1$ for all $x, y \in S$. 
Hint: You may in a suitable way use that the function $\varphi: \mathbb{R}_{0}^{+} \rightarrow \mathbb{R}_{0}^{+}$defined by

$$
\varphi(t)=\frac{t}{1+t} \quad, \quad t \in \mathbb{R}_{0}^{+}
$$

is increasing.

Exercise 1.3. Let $K$ be an arbitrary set, and let $(S, d)$ be a metric space in which $0 \leq d(x, y) \leq 1$ for all $x, y \in S$.

Let $F(K, S)$ denote the set of mappings $f: K \rightarrow S$.

Define $D: F(K, S) \times F(K, S) \rightarrow \mathbb{R}_{0}^{+}$by

$$
D(f, g)=\sup _{t \in K} d(f(t), g(t)) .
$$

1) Show that $D$ is a metric on $F(K, S)$.

2) Let $t_{0} \in K$ be a fixed point in $K$ and define

$$
E v_{t_{0}}: F(K, S) \rightarrow S \quad \text { by } E v_{t_{0}}(f)=f\left(t_{0}\right) .
$$

Show that $E v_{t_{0}}$ is continuous. ( $E v_{t_{0}}$ is called an evaluation mapping.)

Exercise 1.4. (Optional) Exercise 1.1,2) and Exercise 1.3, 2) are both special cases of a general result. Try to formulate such a general result.

\section{Problem Set 2}

Exercise 2.1. Let $(S, d)$ be a metric space. For $x \in S$ and $r \in \mathbb{R}^{+}$, let $B_{r}(x)$ denote the open ball in $S$ with centre $x$ and radius $r$.

Show that the system of open balls in $S$ has the following properties:

1) If $y \in B_{r}(x)$ then $x \in B_{r}(y)$.

2) If $y \in B_{r}(x)$ and $0<s \leq r-d(x, y)$, then $B_{s}(y) \subseteq B_{r}(x)$.

3) If $d(x, y) \geq r+s$, where $x, y \in S$ and $r, s \in \mathbb{R}^{+}$, then $B_{r}(x)$ and $B_{s}(y)$ are mutually disjoint.

Exercise 2.2. Let $(S, d)$ be a metric space. A subset $K$ in $S$ is called bounded in $(S, d)$, if there exists a point $x \in S$ and an $r \in \mathbb{R}^{+}$such that $K \subseteq B_{r}(x)$.

Examine the truth of each of the following three statements:

1) If two subsets $K_{1}$ and $K_{2}$ in $S$ are bounded in $(S, d)$, then their union $K_{1} \cup K_{2}$ is also bounded in $(S, d)$. 
2) If $K \subseteq S$ is bounded in $(S, d)$, then

$$
K^{\prime}=\bigcup_{x \in K}\{y \in S \mid d(x, y) \leq 1\}
$$

is also bounded in $(S, d)$.

3) If $K \subseteq S$ is bounded in $(S, d)$, then

$$
K^{\prime \prime}=\bigcap_{x \in K}\{y \in S \mid d(x, y)>1\}
$$

is also bounded in $(S, d)$.

Exercise 2.3. List all topologies that can be defined on a set $S=\{a, b\}$ containing only two elements $a$ and $b$.

Exercise 2.4. Let $\mathcal{T}$ be the system of subsets $U$ in $\mathbb{R}$ which is of one the following types:

Either (i) $U$ does not contain 0 ,

or (ii) $U$ does contain 0 , and the complementary set $\mathbb{R} \backslash U$ is finite.

1) Show that $\mathcal{T}$ is a topology on $\mathbb{R}$.

2) Show that $\mathbb{R}$ with the tolopology $\mathcal{T}$ is a Hausdorff space.

(A topological space $(S, \mathcal{T})$ is called a Hausdorff space if, for every pair of points $x, y \in S$ with $x \neq y$, there exists a corresponding pair of mutually disjoint, open sets $U, V \in \mathcal{T}$, such that $x \in U$ and $y \in V$.)

3) (Optional) Show that the topology $\mathcal{T}$ on $\mathbb{R}$ does not stem from a metric on $\mathbb{R}$, since there does not exist a countable system of open neighbourhoods of $0 \in \mathbb{R}$ in the topology $\mathcal{T}$ with the property that an arbitrary open neighbourhood of $0 \in \mathbb{R}$ contains a neighbourhood from the system.

\section{Problem Set 3}

Exercise 3.1. Let $S$ be a topological space with topology $\mathcal{T}$, and let $\pi: S \rightarrow \tilde{S}$ be a mapping into a set $\tilde{S}$. Let $\tilde{\mathcal{T}}$ be the quotient topology on $\tilde{S}$ induced from the topology $\mathcal{T}$ on $S$ by the mapping $\pi$.

1) Let $\mathcal{T}^{\prime}$ be a topology on $\tilde{S}$, such that $\pi: S \rightarrow \tilde{S}$ is continuous when $S$ is considered with the topology $\mathcal{T}$ and $\tilde{S}$ with the topology $\mathcal{T}^{\prime}$. Show that $\mathcal{T}^{\prime} \subseteq \tilde{\mathcal{T}}$ 
(The quotient topology $\tilde{\mathcal{T}}$ on $\tilde{S}$ is in other words the 'largest' topology on $\tilde{S}$ for which $\pi: S \rightarrow \tilde{S}$ is continuous.)

2) Show that when $\tilde{S}$ has the quotient topology determined by the mapping $\pi: S \rightarrow \tilde{S}$, then the following holds:

A mapping $f: \tilde{S} \rightarrow T$ into a topological space $T$ is continuous if and only if the composite mapping $f \circ \pi: S \rightarrow T$ is continuous.

Exercise 3.2. Let $S$ be a topological space. For every pair of real-valued functions $f, g: S \rightarrow \mathbb{R}$, we can in the usual way define the functions $f+g$, $f-g, f \cdot g$, and (if $g(x) \neq 0$ for all $x \in S$ ) $f / g$.

1) Show that if $f$ and $g$ are continuous at a point $x_{0} \in S$, then also $f+g, f-g, f \cdot g$, and (when it is defined) $f / g$ are continuous at $x_{0} \in S$. (Carry through the argument in at least one case.)

2) Assume that $f, g: S \rightarrow \mathbb{R}$ are continuous. Show that $U=\{x \in S \mid f(x)<g(x)\}$ is an open set in $S$.

3) Let $f_{1}, \ldots, f_{k}: S \rightarrow \mathbb{R}$ be contiuous real-valued functions and $a_{1}, \ldots, a_{k} \in \mathbb{R}$ real numbers. Show that $U=\left\{x \in S \mid f_{i}(x)<a_{i}, i=1, \ldots, k\right\}$ is an open set in $S$.

Exercise 3.3. Let $S$ be a topological space with topology $\mathcal{T}$, and let $A$ be an arbitrary subset in $S$. Equip $A$ with the induced topology $\mathcal{T}_{A}$.

Show that a subset $B^{\prime} \subseteq A$ is closed in $A$ with the topology $\mathcal{T}_{A}$ if and only if there exists a closed subset $B \subseteq S$ in the topology $\mathcal{T}$ such that $B^{\prime}=A \cap B$.

Exercise 3.4. Let $f: X \rightarrow Y$ be a mapping between topological spaces $X$ and $Y$.

If $f: X \rightarrow Y$ maps a subset $X^{\prime} \subseteq X$ in $X$ into a subset $Y^{\prime} \subseteq Y$ in $Y$, then $f$ determines a mapping $f^{\prime}: X^{\prime} \rightarrow Y^{\prime}$ defined by $f^{\prime}(x)=f(x)$ for $x \in X^{\prime}$.

When a subset of a topological space is considered as a topological space in the following, it is always with the induced topology.

1) Let $f^{\prime}: X^{\prime} \rightarrow Y^{\prime}$ be a mapping determined by $f: X \rightarrow Y$ as above. Show that if $f$ is continuous, then $f^{\prime}$ is continuous.

2) Let $A_{1}$ and $A_{2}$ be closed subsets in $X$ such that $X=A_{1} \cup A_{2}$. Let $f_{1}: A_{1} \rightarrow Y$ and $f_{2}: A \rightarrow Y$ be the mappings determined by $f$, i.e. the restrictions of $f$ to $A_{1}$ and $A_{2}$, respectively.

Show that if $f_{1}$ and $f_{2}$ are continuous, then $f$ is continuous. 


\section{Problem Set 4}

Exercise 4.1. Let $W_{1}$ and $W_{2}$ be arbitrary subsets in the topological space $S$. Show that

1) $\operatorname{int}\left(W_{1} \cap W_{2}\right)=\operatorname{int} W_{1} \cap \operatorname{int} W_{2}$.

$2) \operatorname{int}\left(W_{1} \cup W_{2}\right) \supseteq \operatorname{int} W_{1} \cup \operatorname{int} W_{2}$.

Give an example that the equality sign in 2 ) does not apply in general.

Exercise 4.2. Show that a topological space $S$ is a $\mathrm{T}_{1}$-space if and only if every subset in $S$ containing exactly one point is a closed subset.

Exercise 4.3. Let $S$ be a Hausdorff space, and let $W$ be an arbitrary subset in $S$. (It is sufficient that $S$ satisfies the separation property $\mathrm{T}_{1}$.)

Show that if $x \in S$ is an accumulation point of $W$, then every neighbourhood of $x$ in $S$ contains infinitely many mutually different points from $W$.

Exercise 4.4. Let $(S, d)$ be a metric space. For an arbitrary non-empty subset $W$ in $S$, we define a function $\varphi: S \rightarrow \mathbb{R}$ by

$$
\varphi(x)=\inf \{d(x, y) \mid y \in W\} \quad \text { for } \quad x \in S .
$$

We call $\varphi(x)$ the distance from $x$ to $W$, and write, accordingly, $\varphi(x)=d(x, W)$.

1) Let $x_{1}, x_{2} \in S$ be arbitrary points in $S$.

First show that for an arbitrary point $y \in W$ it holds that

$$
\varphi\left(x_{1}\right) \leq d\left(x_{1}, x_{2}\right)+d\left(x_{2}, y\right)
$$

Next show that

$$
\left|\varphi\left(x_{1}\right)-\varphi\left(x_{2}\right)\right| \leq d\left(x_{1}, x_{2}\right),
$$

and conclude from this that $\varphi$ is (uniformly) continuous on $S$.

2) Show that

$$
d(x, W)=\varphi(x)=0 \Longleftrightarrow x \in \bar{W},
$$

where $\bar{W}$ as usual denotes the closure of $W$.

3) (Optional) Let $A_{1}$ and $A_{2}$ be disjoint, non-empty closed subsets in the metric space $(S, d)$. Show that there exist disjoint, open sets $U_{1}$ and $U_{2}$ in $S$, such that $A_{1} \subseteq U_{1}$ and $A_{2} \subseteq U_{2}$.

Hint: Consider the distance functions $\varphi_{1}(x)=d\left(x, A_{1}\right)$ and $\varphi_{2}(x)=d\left(x, A_{2}\right)$.

Exercise 4.5. Let $S=\{x \in \mathbb{R} \mid 0 \leq x<1\}$. Consider the family of subsets $\mathcal{T}$ in $S$ consisting of the empty set $\emptyset$ and every subset $U \subseteq S$ of the form $U=\{x \in \mathbb{R} \mid 0 \leq x<k\}$ for a number $k$ with $0<k \leq 1$. 
1) Show that $\mathcal{T}$ is a topology on $S$.

2) Show that in the topological space $(S, \mathcal{T})$, the sequence $\left(x_{n}=\frac{1}{n+1}\right)$ will have every point in $S$ as limit point.

3) Examine if the topology $\mathcal{T}$ stems from a metric on $S$.

\section{Problem Set 5}

Exercise 5.1. Deduce the existence of a supremum from the principle of nested intervals.

Exercise 5.2. Let $S$ be a topological space, and let $\left(f_{n}\right)$, or in more detail $f_{1}, f_{2}, \ldots, f_{n}, \ldots$, be a sequence of continuous functions $f_{n}: S \rightarrow \mathbb{R}$, such that for all $x \in S$ it holds that

$$
\begin{aligned}
& \text { (i) } f_{n}(x) \geq 0 \\
& \text { (ii) } f_{1}(x) \geq f_{2}(x) \geq \cdots \geq f_{n}(x) \geq \ldots \\
& \text { (iii) } \lim _{n \rightarrow \infty} f_{n}(x)=0
\end{aligned}
$$

In other words: The decreasing sequence of functions $\left(f_{n}\right)$ converges pointwise to the 0 -function.

For $\varepsilon>0$ and $n \in \mathbb{N}$ we set

$$
U_{n}(\varepsilon)=\left\{x \in S \mid 0 \leq f_{n}(x)<\varepsilon\right\} .
$$

1) Show that $U_{n}(\varepsilon)$ is an open set in $S$.

2) Show that for fixed $\varepsilon>0$, the collection of sets $\left\{U_{n}(\varepsilon) \mid n \in \mathbb{N}\right\}$ defines an open covering of $S$.

3) Now assume that $S$ is compact. Show then that for every $\varepsilon>0$ there is an $n_{0} \in \mathbb{N}$, such that for all $n \geq n_{0}$ it holds that

$$
0 \leq f_{n}(x)<\varepsilon \text { for all } x \in S
$$

or written with quantifiers,

$$
\forall \varepsilon>0 \exists n_{0} \in \mathbb{N} \forall n \in \mathbb{N}: n \geq n_{0} \Rightarrow \forall x \in S: 0 \leq f_{n}(x)<\varepsilon .
$$

We conclude that, under the given assumptions, the sequence of functions $\left(f_{n}\right)$ converges uniformly to the 0 -function. 
This result is due to the Italian mathematician Ulisse Dini (1845-1918) and is known as Dini's Theorem.

4) (Optional) Is it of importance that $S$ is compact in 3 )?

Exercise 5.3. Let $f, g:[a, b] \rightarrow \mathbb{R}$ be continuous functions defined in a closed and bounded interval $[a, b]$. Assume that $f(x)<g(x)$ for every $x \in[a, b]$.

Show that

$$
K=\left\{(x, y) \in \mathbb{R}^{2} \mid a \leq x \leq b, f(x) \leq y \leq g(x)\right\}
$$

is a compact subset in $\mathbb{R}^{2}$.

Exercise 5.4. Let $K_{1} \supseteq K_{2} \supseteq \cdots \supseteq K_{n} \supseteq \ldots$ be a descending sequence of non-empty, compact subsets in a Hausdorff space $S$.

Show that the intersection of sets $\bigcap_{n=1}^{\infty} K_{n}$ is non-empty.

Exercise 5.5. Let $S=\mathbb{N} \cup\{0\}$ be the set of non-negative integers.

For every natural number $n \in \mathbb{N}$ we define a subset $U_{n}$ in $S$ by

$$
U_{n}=\{n \cdot p \in S \mid p=0,1,2, \ldots\} \text {. }
$$

1) Show that for all $n, m \in \mathbb{N}$, the intersection $U_{n} \cap U_{m}$ has the form $U_{k}$ for a suitable $k \in \mathbb{N}$.

Consider the family $\mathcal{T}$ of subsets in $S$ which consists of the empty set $\emptyset$ and all subsets $U$ in $S$ that can be written as a union of sets from $\left\{U_{n} \mid n \in \mathbb{N}\right\}$, i.e. $U=\cup_{\alpha \in A} U_{n_{\alpha}}$.

2) Show that $\mathcal{T}$ is a topology on $S$. (The system of subsets $\left\{U_{n} \mid n \in \mathbb{N}\right\}$ in $S$ is called a basis for the topology $\mathcal{T}$.)

3) Show that the sequence $\left(x_{n}=n !\right)$ will converge to every point in the topological space $(S, \mathcal{T})$.

\section{Problem Set 6}

Exercise 6.1. Let $S$ be a Hausdorff space. A function $f: S \rightarrow \mathbb{R}$ is said to be lower semicontinuous, if the following condition is satisfied:

For every $x \in S$ and every $\varepsilon>0$ there exists a neighbourhood $N$ of $x$ in $S$, such that

$$
f(x)-\varepsilon<f(y) \text { for } y \in N \text {. }
$$


1) Show that a lower semicontinuous function $f: S \rightarrow \mathbb{R}$ is bounded from below on every sequentially compact subset $K$ in $S$.

Hint: You can prove this indirectly, taking the proof of Theorem 1.10.5, page 35 (the first proof) as your source of inspiration.

2) Show that a lower semicontinuous function $f: S \rightarrow \mathbb{R}$ assumes a minimum value on every sequentially compact subset $K$ in $S$.

Hint: Construct a sequence $\left(x_{n}\right)$ in $K$ for which

$$
\lim _{n \rightarrow \infty} f\left(x_{n}\right)=\inf f(K)
$$

and make use of this to determine a point $x_{0} \in K$, such that $f\left(x_{0}\right)=\inf f(K)$. (In a suitable setting this is the so-called direct method in the calculus of variations, and the sequence $\left(x_{n}\right)$ is called a minimizing sequence.)

Exercise 6.2. Let $f: \mathbb{R} \rightarrow \mathbb{R}$ be a differentiable function with bounded differential quotient. Show that $f$ is uniformly continuous.

Hint: You can use the classical Mean Value Theorem.

Exercise 6.3. A subset $K$ in a metric space $(S, d)$ is called precompact if for every $\epsilon>0$ there exist finitely many points $x_{1}, \ldots, x_{p} \in K$ such that $K \subseteq B_{\varepsilon}\left(x_{1}\right) \cup \cdots \cup B_{\varepsilon}\left(x_{p}\right)$.

1) Show that a subset $K \subseteq \mathbb{R}^{n}$ in the space $\mathbb{R}^{n}$ (with Euclidean metric) is precompact if and only if it is bounded.

2) Let $f: X \rightarrow Y$ be a mapping between the metric spaces $\left(X, d_{X}\right)$ and $\left(Y, d_{Y}\right)$, and let $K \subseteq X$ be a precompact subset in $X$. Show that if $f$ is uniformly continuous in $K$, then the image set $f(K) \subseteq Y$ is precompact in $Y$.

Exercise 6.4. Let $T$ be a point set with more than one element equipped with the discrete topology.

1) Show that a topological space $S$ is connected if and only if every continuous mapping $f: S \rightarrow T$ is constant.

2) Let $\left\{W_{i} \mid i \in I\right\}$ be a family of connected subsets in a topological space $S$, such that for every pair of sets $W_{i}$ and $W_{j}$ from the family it holds that $W_{i} \cap W_{j} \neq \emptyset$. Then show that the union $\bigcup_{i \in I} W_{i}$ is a connected subset in $S$.

Exercise 6.5. Prove the following theorem: Let $M$ be an arbitrary subset in the number space $\mathbb{R}^{k}$ with the usual topology, and let $\left\{U_{i} \mid i \in I\right\}$ be an arbitrary system of open sets in $\mathbb{R}^{k}$ that covers $M$. Then, either there exists a finite subsystem $\left\{U_{i_{1}}, \ldots, U_{i_{n}}\right\}$, or, there exists a countable subsystem $\left\{U_{i_{1}}, U_{i_{2}}, \ldots, U_{i_{n}}, \ldots\right\}$ that covers $M$.

The theorem is due to the Finnish mathematician Ernst Lindelöf (18701946) and is known as Lindelöf's covering theorem. 
Hint: Consider the following system of open balls in $\mathbb{R}^{k}$ :

$\left\{B_{r}(x) \mid r \in \mathbb{R}^{+}\right.$is rational; $x \in \mathbb{R}^{k}$ has rational coordinates $\}$.

You may utilize without proof that this system is countable (can be assigned numbers).

\section{Problem Set 7}

Exercise 7.1. Let $E$ be a subset in the topological space $S$. Show that if $E$ is connected, then the closure $\bar{E}$ of $E$ is also connected.

Exercise 7.2. Let $(S, d)$ be a metric space. For an arbitrary pair of non-empty subsets $A$ and $B$ in $S$, we define the distance from $A$ to $B$, denoted $d(A, B)$, by

$$
d(A, B)=\inf \{d(x, y) \mid x \in A, y \in B\} .
$$

1) As in Exercise 4.4 we define for every $x \in S$ the distance from $x$ to $B$ by

$$
d(x, B)=\inf \{d(x, y) \mid y \in B\} .
$$

Argue that for arbitrary points $x \in A$ and $y \in B$ it holds that

$$
d(A, B) \leq d(x, B) \text { and } \inf \left\{d\left(x^{\prime}, B\right) \mid x^{\prime} \in A\right\} \leq d(x, y) .
$$

Utilize this to show that

$$
d(A, B)=\inf \{d(x, B) \mid x \in A\} .
$$

2) Show that if $A$ is compact, then there exists a point $a_{0} \in A$ such that $d(A, B)=d\left(a_{0}, B\right)$.

Hint: You can use that the function $\varphi: S \rightarrow \mathbb{R}$, defined by $\varphi(x)=d(x, B)$, is continuous.

Next show that if $B$ is also compact, then there exists a point $b_{0} \in B$ such that $d(A, B)=d\left(a_{0}, b_{0}\right)$.

3) (Optional) Let $K$ be a compact subset in $S$ contained in the open set $U$ in $S$, i.e. $K \subseteq U \subseteq S$. Show that there exists an $r \in \mathbb{R}^{+}$, such that $B_{r}(x) \subseteq U$ for every $x \in K$.

Exercise 7.3. Let $E=C^{\infty}([0,2 \pi], \mathbb{R})$ be the vector space of differentiable functions $f:[0,2 \pi] \rightarrow \mathbb{R}$ of class $C^{\infty}$. 
For $f \in E$ we set:

$$
\begin{aligned}
& \|f\|_{0}=\sup \{|f(x)| \mid x \in[0,2 \pi]\} \\
& \|f\|_{1}=\sup \left\{\left|f^{\prime}(x)\right|+\left|f^{\prime}(x)\right| \mid x \in[0,2 \pi]\right\} .
\end{aligned}
$$

1) Show that $\|\cdot\|_{0}$ and $\|\cdot\|_{1}$ are norms in $E$.

Define the linear mapping $D: E \rightarrow E$ by associating to $f \in E$ the derivative $f^{\prime} \in E$ of $f$, i.e.

$$
D(f)=f^{\prime} \text { for } f \in E .
$$

2) Show that for every $n \in \mathbb{N}$ there exists a function $f_{n} \in E$ for which $\left\|f_{n}\right\|_{0}=1$ and $\left\|D\left(f_{n}\right)\right\|_{0}=n$.

Utilize this to show that $D: E \rightarrow E$ is not continuous, when $E$ is equipped with the norm $\|\cdot\|_{0}$.

3) Show that $D: E_{1} \rightarrow E_{0}$ is continuous, when $E_{1}$ is $E$ equipped with the norm $\|\cdot\|_{1}$, and $E_{0}$ is $E$ equipped with the norm $\|\cdot\|_{0}$.

\section{Problem Set 8}

Exercise 8.1. Let $V$ be the space of continuous functions $f: \mathbb{R} \rightarrow \mathbb{R}$, such that $f(x) \rightarrow 0$ for $|x| \rightarrow \infty$.

For a function $f \in V$ holds, in other words

$$
\forall \varepsilon>0 \exists a \in \mathbb{R}^{+} \forall x \in \mathbb{R}:|x|>a \Rightarrow|f(x)|<\varepsilon .
$$

Define the operations 'addition' and 'multiplication with scalars' in $V$ by the obvious pointwise definitions.

1) Show that $V$ is a vector space.

2) Show that every function $f \in V$ is bounded.

Making use of 2), we can define

$$
\|f\|=\sup \{|f(x)| \mid x \in \mathbb{R}\} \text { for } f \in V .
$$

3) Show that $\|\cdot\|$ is a norm in $V$.

Exercise 8.2. Let $V$ be the space of sequences

$$
x=\left(\alpha_{1}, \alpha_{2}, \ldots, \alpha_{i}, \ldots\right)
$$

of real numbers $\alpha_{i} \in \mathbb{R}$ in which at most finitely many $\alpha_{i} \neq 0$. 
Define the operations 'addition' and 'multiplication with scalars' in $V$ by the obvious coordinate-wise definitions. Further, set

$$
\|x\|=\sum_{i=1}^{\infty}\left|\alpha_{i}\right| .
$$

1) Show that $V$ is a vector space and that $\|\cdot\|$ is a norm in $V$.

2) Consider an infinite series of real numbers,

$$
\sum_{i=1}^{\infty} a_{i} .
$$

(There is no condition that at most finitely many $a_{i} \neq 0$.)

Define the sequence $\left(x_{n}\right)$ in $V$ by $x_{1}=\left(a_{1}, 0,0, \ldots\right), x_{2}=\left(a_{1}, a_{2}, 0, \ldots\right)$, and, in general,

$$
x_{n}=\left(a_{1}, a_{2}, \ldots, a_{n}, 0, \ldots\right) .
$$

Show that the series $\sum_{i=1}^{\infty}\left|a_{i}\right|$ is convergent, if and only if the sequence $\left(x_{n}\right)$ is a Cauchy sequence (fundamental sequence) in the normed vector space $V$, i.e.

$$
\forall \varepsilon>0 \exists n_{0} \in \mathbb{N} \forall n, k \in \mathbb{N}: n \geq n_{0} \Rightarrow\left\|x_{n+k}-x_{n}\right\|<\varepsilon .
$$

3) Give an example of a Cauchy sequence in the normed vector space $V$ that has no limit point in $V$.

Exercise 8.3. Let $V$ be a finite dimensional, normed vector space with norm $\|\cdot\|$. Let $L: V \rightarrow V$ be an arbitrary linear mapping. Show that there exists a unit vector $x_{0} \in V$, i.e. $\left\|x_{0}\right\|=1$, such that $\left\|L\left(x_{0}\right)\right\|=\|L\|$, where $\|L\|$ is the operator norm of $L$, i.e. $\|L\|=\sup \{\|L(x)\| \mid\|x\|=1\}$.

(Optional) Show by an example that this does not hold in general, when $V$ has infinite dimension.

\section{Problem Set 9}

Exercise 9.1. Let $C([0,1], \mathbb{R})$ be the vector space of continuous real-valued functions in the unit interval $[0,1]$. For a continuous function $f:[0,1] \rightarrow \mathbb{R}$ we set

$$
\|f\|_{1}=\int_{0}^{1}|f(x)| d x .
$$

1) Show that $\|\cdot\|_{1}$ is a norm in $C([0,1], \mathbb{R})$. 
We now equip $C([0,1], \mathbb{R})$ as a normed vector space with the norm $\|\cdot\|_{1}$ and define the function

$$
I: C([0,1], \mathbb{R}) \rightarrow \mathbb{R} \text { by } I(f)=\int_{0}^{1} f(x) d x .
$$

2) Show that $I$ is a continuous linear function.

3) Determine the operator norm of $I$.

4) In $C([0,1], \mathbb{R})$ equipped with the norm $\|\cdot\|_{1}$, consider the sequences $\left(f_{n}\right)$ and $\left(g_{n}\right)$ defined by

$$
\begin{aligned}
& f_{n}(x)= \begin{cases}1-n x & \text { for } 0 \leq x \leq \frac{1}{n} \\
0 & \text { for } \frac{1}{n} \leq x \leq 1\end{cases} \\
& g_{n}(x)= \begin{cases}n-n^{2} x & \text { for } 0 \leq x \leq \frac{1}{n} \\
0 & \text { for } \frac{1}{n} \leq x \leq 1\end{cases}
\end{aligned}
$$

Examine the convergence of each of these sequences and, in the case of convergence, determine the limit function.

Exercise 9.2. Let $f: E \rightarrow F$ be a mapping between normed vector spaces $E$ and $F$ which is differentiable at $0 \in E$ and has the property $f(\alpha h)=\alpha f(h)$ for all $\alpha \in \mathbb{R}$ and all $h \in E$. Show that $f$ is linear.

Exercise 9.3. Let $C([0,1], \mathbb{R})$ be the vector space of continuous functions $f:[0,1] \rightarrow \mathbb{R}$ equipped as a normed vector space with the norm

$$
\|f\|=\sup _{0 \leq x \leq 1}|f(x)| .
$$

Let $\Phi=\Phi(x, y):[0,1] \times[0,1] \rightarrow \mathbb{R}$ be a continuous function in two variables defined in the square $[0,1] \times[0,1]$ in $\mathbb{R}^{2}$. Assume that $\Phi(x, y) \geq 0$ for all $(x, y) \in[0,1] \times[0,1]$.

Define the function $\varphi=\varphi(x):[0,1] \rightarrow \mathbb{R}$ by

$$
\varphi(x)=\sup _{0 \leq y \leq 1} \Phi(x, y) .
$$

For $f \in C([0,1], \mathbb{R})$ we define the function $f_{\Phi}=f_{\Phi}(y):[0,1] \rightarrow \mathbb{R}$ by

$$
f_{\Phi}(y)=\int_{0}^{1} \Phi(x, y) f(x) d x
$$

1) Show that for every $\varepsilon>0$ there exists a $\delta>0$ such that 
Exercises

$$
\begin{aligned}
& \text { a) } \quad\left(x_{0}, y\right)-\varepsilon \leq \Phi(x, y) \leq \Phi\left(x_{0}, y\right)+\varepsilon \\
& \text { for }\left|x-x_{0}\right| \leq \delta \text { and all } y \in[0,1] \\
& \text { b) }\left|\Phi(x, y)-\Phi\left(x, y_{0}\right)\right| \leq \varepsilon \\
& \text { for }\left|y-y_{0}\right| \leq \delta \text { and all } x \in[0,1]
\end{aligned}
$$

Make use of this to show that the functions $\varphi=\varphi(x)$ and $f_{\Phi}=f_{\Phi}(y)$ are continuous.

Since $f_{\Phi} \in C([0,1], \mathbb{R})$, we can define the mapping

$$
L: C([0,1], \mathbb{R}) \text { by } L(f)=f_{\Phi} .
$$

The mapping $L$ is called an integral operator with kernel $\Phi$.

2) Show that $L$ is a continuous linear mapping.

3) Show that

$$
\|L(f)\| \leq\left(\int_{0}^{1} \varphi(x) d x\right)\|f\|
$$

for all $f \in C([0,1], \mathbb{R})$.

4) Show that the operator norm for $L$ is given by

$$
\|L\|=\sup _{0 \leq y \leq 1} \int_{0}^{1} \Phi(x, y) d x
$$

\section{Problem Set 10}

Exercise 10.1. Let $U$ be an open set in the normed vector space $E$. A realvalued function $f: U \rightarrow \mathbb{R}$ is said to have a local maximum (minimum) at a point $x_{0} \in U$ if there exists a neighbourhood $N \subseteq U$ of $x_{0}$ such that $f(x) \leq f\left(x_{0}\right) \quad\left(f(x) \geq f\left(x_{0}\right)\right)$ for all $x \in N$.

1) Suppose that the function $f: U \rightarrow \mathbb{R}$ is differentiable at the point $x_{0} \in U$. Prove that for each fixed $h \in E$, there exists an $r>0$ such that the function $g(t)=f\left(x_{0}+t h\right)$ is defined for $\left.t \in\right]-r, r[$ and is differentiable at 0 with derivative $g^{\prime}(0)=D f\left(x_{0}\right)(h)$. 
2) Suppose that the function $f: U \rightarrow \mathbb{R}$ is differentiable at the point $x_{0} \in U$ and that $f$ has a local maximum (minimum) at $x_{0} \in U$. Prove that the differential of $f$ at $x_{0}$ is zero, i.e. $D f\left(x_{0}\right)=0$.

Exercise 10.2. Let $\mathcal{H}$ denote a vector space with inner product $\langle\cdot, \cdot\rangle$ and associated norm $\|\cdot\|$ defined by $\|x\|=\sqrt{\langle x, x\rangle}$ for $x \in \mathcal{H}$. (Example: $\mathcal{H}=\mathbb{R}^{n}$ equipped with the standard inner product $\langle x, y\rangle=\sum_{i=1}^{n} x_{i} y_{i}$.)

Let $E$ denote a finite dimensional proper subspace of $\mathcal{H}$ and let $u \in \mathcal{H}$ be a fixed point in $\mathcal{H}$ outside $E$.

Define the function $f: E \rightarrow \mathbb{R}$ by

$$
f(x)=\|x-u\|^{2}=\langle x-u, x-u\rangle \text { for } x \in E .
$$

1) Prove that $f$ is differentiable at every point $x \in E$ with differential $D f(x): E \rightarrow \mathbb{R}$ given by

$$
D f(x)(h)=2\langle x-u, h\rangle \text { for all } h \in E .
$$

2) Prove that the differential of $f$ is zero at exactly one point $x_{0} \in E$.

Hint: The differential of $f$ is zero at $x_{0} \in E$, i.e. $D f\left(x_{0}\right)=0$, if and only if the vector $x_{0}-u$ is orthogonal to $E$.

Exercise 10.3. Let $\mathbb{R}^{n}$ denote $n$-dimensional Euclidean space equipped with the usual inner product

$$
\langle x, y\rangle=\sum_{i=1}^{n} x_{i} y_{i}
$$

and the associated norm

$$
\|x\|=\sqrt{\langle x, x\rangle}=\sqrt{\sum_{i=1}^{n} x_{i} x_{i}} .
$$

Denote by $E=C^{\mathbf{l}}\left([a, b], \mathbb{R}^{n}\right)$ the space of differentiable real-valued functions $f:[a, b] \rightarrow \mathbb{R}^{n}$ of class $C^{1}$ defined on the interval $[a, b]$ in $\mathbb{R}$. We can equip $E$ with the structure of a normed vector space (proof not required) with norm

$$
\|f\|_{1}=\sup _{a \leq t \leq b}\left(\|f(t)\|+\left\|f^{\prime}(t)\right\|\right) .
$$

Define the (kinetic) energy function $K: E \rightarrow \mathbb{R}$ by

$$
\left.K(f)=\frac{1}{2} \int_{a}^{b} \| f^{\prime} t\right) \|^{2} d t=\frac{1}{2} \int_{a}^{b}\left\langle f^{\prime}(t), f^{\prime}(t)\right\rangle d t \quad \text { for } f \in E .
$$


1) Prove that $K$ is differentiable at every $f \in E$ with differential $D K(f): E \rightarrow \mathbb{R}$ given by

$$
D K(f)(h)=\int_{a}^{b}\left\langle f^{\prime}(t), h^{\prime}(t)\right\rangle d t \quad \text { for all } h \in E .
$$

2) Prove that the differential of $K$ at $f \in E$ is zero, i.e. $D K(f)=0$, if and only if $f$ is a constant function. (Hint: Try to set $h=f$.)

3)(Optional) Provide a physical interpretation of the result in 2).

\section{Problem Set 11}

Exercise 11.1. Let $(S, d)$ be a metric space. Show that if a subset $A$ in $S$ is a complete metric space in the induced metric from $S$, then $A$ is a closed set in $S$.

Exercise 11.2. Let $X$ be a compact topological space, and let $S$ be a complete metric space with metric $d$.

By $C(X, S)$ we denote the space of continuous mappings $f: X \rightarrow S$.

For $f, g \in C(X, S)$ we put

$$
D(f, g)=\sup _{x \in X} d(f(x), g(x)) .
$$

Then $D$ is a metric in $C(X, S)$. (Proof not required.)

1) Show that $\left(f_{n}\right)$ is a Cauchy sequence in the metric space $(C(X, S), D)$ if and only if $\left(f_{n}: X \rightarrow S\right)$ is a uniform Cauchy sequence, i.e.

$\forall \varepsilon>0 \exists n_{0} \in \mathbb{N} \forall n, m \in \mathbb{N}: n, m \geq n_{0} \Longrightarrow \forall x \in X: d\left(f_{n}(x), f_{m}(x)\right)<\varepsilon$

Now let $\left(f_{n}\right)$ be a Cauchy sequence in $(C(X, S), D)$.

2) Show that for every $x \in X$, there exists a uniquely determined $y \in S$, such that $f_{n}(x) \rightarrow y$ for $n \rightarrow \infty$.

Define a mapping $f: X \rightarrow S$ by setting $f(x)=y$ for all $x \in X$, where $y \in S$ is determined as in 2). In other words, the mapping $f$ is defined by

$$
f(x)=\lim _{n \rightarrow \infty} f_{n}(x) \quad \text { for } \quad x \in X .
$$

3) First show that $\left(f_{n}\right)$ converges uniformly to $f$ for $n$ going to $\infty$, i.e.

$$
\forall \varepsilon>0 \exists n_{0} \in \mathbb{N} \forall n \in \mathbb{N}: n \geq n_{0} \Longrightarrow \forall x \in X: d\left(f_{n}(x), f(x)\right)<\varepsilon .
$$


Next show that $f: X \rightarrow S$ is continuous.

Hint:

$$
d\left(f(x), f\left(x_{0}\right)\right) \leq d\left(f(x), f_{n}(x)\right)+d\left(f_{n}(x), f_{n}\left(x_{0}\right)\right)+d\left(f_{n}\left(x_{0}\right), f\left(x_{0}\right)\right) .
$$

4) Show that $(C(X, S), D)$ is a complete metric space.

\section{Problem Set 12}

Exercise 12.1. (Local Existence and Uniqueness Theorem for Autonomous Ordinary Differential Equations.)

Let $E$ be a Banach space, and let $U \subseteq E$ be an open set in $E$. The norm in $E$ is denoted by $\|\cdot\|$.

A mapping $f: U \rightarrow E$ is said to be Lipschitz continuous in $U$, if there exists a constant $k$, such that

$$
\left\|f\left(x_{1}\right)-f\left(x_{2}\right)\right\| \leq k\left\|x_{1}-x_{2}\right\| \text { for all } x_{1}, x_{2} \in U .
$$

Now assume that $f: U \rightarrow E$ is a Lipschitz continuous mapping in $U$. (One can think of $f$ as a vector field in $U$ by placing the vector $f(x) \in E$ at every point $x \in U$.)

Consider the Initial Value Problem consisting of the differential equation (i) below together with the initial value condition (ii):

$$
\text { (i) } \frac{d x}{d t}=f(x) \quad \text { (ii) } x(0)=x_{0} \in U \text {. }
$$

By a solution, or, an integral curve, to the Initial Value Problem (i) and (ii) we understand a differentiable curve $\varphi: J \rightarrow U$ defined in an interval $J$ around $0 \in \mathbb{R}$, such that

$$
\frac{d \varphi}{d t}=f(\varphi(t)) \text { for all } t \in J
$$

and such that $\varphi(0)=x_{0}$.

1) Show that $\varphi: J \rightarrow U$ solves (i) and (ii) if and only if $\varphi$ satisfies the integral equation

$$
\varphi(t)=x_{0}+\int_{0}^{t} f(\varphi(\tau)) d \tau
$$

For $a>0$, let $J_{a}$ denote the interval $J_{a}=[-a, a]$, and for $b>0$, let $S_{b}=\left\{x \in E\|\| x-x_{0} \| \leq b\right\}$ denote the closed ball in $E$ with centre $x_{0} \in U$ 
and radius $b$. For $b>0$ sufficiently small, we have $S_{b} \subseteq U$, and we shall only consider such $b$.

Let $C\left(J_{a}, S_{b}\right)$ denote the space of continuous mappings $\varphi: J_{a} \rightarrow S_{b}$ equipped with the metric $D$ as in Exercise 11.2.

To $\varphi \in C\left(J_{a}, S_{b}\right)$ we associate $\psi: J_{a} \rightarrow E$ defined by

$$
\psi(t)=x_{0}+\int_{0}^{t} f(\varphi(\tau)) d \tau \quad \text { for } \quad t \in J_{a}
$$

2) Show that for sufficiently small $a>0$, the mapping $\psi \in C\left(J_{a}, S_{b}\right)$.

3) Show that for sufficiently small $a>0$, the mapping

$$
T: C\left(J_{a}, S_{b}\right) \rightarrow C\left(J_{a}, S_{b}\right),
$$

which assigns $\psi=T(\varphi) \in C\left(J_{a}, S_{b}\right)$ to $\varphi \in C\left(J_{a}, S_{b}\right)$, is a contraction.

4) Show that with $a>0$ as in 3 ), there exists a unique solution $\varphi \in$ $C\left(J_{a}, S_{b}\right)$ to the differential equation

$$
\frac{d x}{d t}=f(x),
$$

such that $\varphi(0)=x_{0}$.

5) Show that if $\varphi_{1}: J_{1} \rightarrow U$ and $\varphi_{2}: J_{2} \rightarrow U$ are two solutions to the differential equation

$$
\frac{d x}{d t}=f(x)
$$

defined in overlapping open intervals $J_{1}$ and $J_{2}$ in $\mathbb{R}$, such that $\varphi_{1}\left(t_{0}\right)=\varphi_{2}\left(t_{0}\right)$ at a point $t_{0} \in J_{1} \cap J_{2}$, then $\varphi_{1}(t)=\varphi_{2}(t)$ at all points $t \in J_{1} \cap J_{2}$.

6) Show that there exists a unique maximal solution to the Initial Value Problem (i) and (ii). (A maximal solution is a solution with an open interval of definition that cannot be extended.)

\section{Problem Set 13}

Exercise 13.1. Let $U \subseteq E$ and $V \subseteq F$ be open sets in the Banach spaces $E$ and $F$. Let $f: U \rightarrow V$ be a differentiable mapping of class $C^{r}, r \geq 1$. Assume that

(i) $f: U \rightarrow V$ is bijective.

(ii) $D f(x): E \rightarrow F$ is a toplinear isomorphism for all $x \in U$. 
Show that $f: U \rightarrow V$ is a diffeomorphism of class $C^{r}$.

Exercise 13.2. Let $E_{1}, E_{2}$ and $F$ be normed vector spaces. We consider, as usual, $E_{1} \times E_{2}$ as a normed vector space with the maximum norm.

Let $U \subseteq E_{1} \times E_{2}$ be an open set, and let $\left(x_{1}, x_{2}\right) \in U$ be an arbitrarily chosen point in $U$, kept fixed in the following.

Consider a mapping $f: U \rightarrow F$ which is differentiable at $\left(x_{1}, x_{2}\right) \in U$.

The restriction $f \mid\left(E_{1} \times\left\{x_{2}\right\}\right) \cap U$ of $f$ to $\left(E_{1} \times\left\{x_{2}\right\}\right) \cap U$ can be considered as a mapping of an open set in $E_{1}$ into $F$. This mapping is differentiable at $x_{1}$, and therefore has a continuous linear mapping $D_{E_{1}} f\left(x_{1}, x_{2}\right): E_{1} \rightarrow F$ as differential. We call $D_{E_{1}} f\left(x_{1}, x_{2}\right)$ the partial differential of $f$ along $E_{1}$ at $\left(x_{1}, x_{2}\right) \in U$. We have in other words,

$$
D_{E_{1}} f\left(x_{1}, x_{2}\right)=D\left(f \mid\left(E_{1} \times\left\{x_{2}\right\}\right) \cap U\right)\left(x_{1}\right) .
$$

Similarly, we can define the partial differential $D_{E_{2}} f\left(x_{1}, x_{2}\right)$ of $f$ along $E_{2}$ at $\left(x_{1}, x_{2}\right) \in U$.

1) In case $E_{1}=\mathbb{R}^{n}, E_{2}=\mathbb{R}^{m}$ and $F=\mathbb{R}^{k}$, find the matrix descriptions of the partial differentials $D_{E_{1}} f\left(x_{1}, x_{2}\right)$ and $D_{E_{2}} f\left(x_{1}, x_{2}\right)$ with respect to the canonical bases in $\mathbb{R}^{n}, \mathbb{R}^{m}$ and $\mathbb{R}^{k}$.

2) Show that the differential $D f\left(x_{1}, x_{2}\right): E_{1} \times E_{2} \rightarrow F$ of $f: U \rightarrow F$ at $\left(x_{1}, x_{2}\right) \in U$ is given by

$$
D F\left(x_{1}, x_{2}\right) \cdot\left(h_{1}, h_{2}\right)=D_{E_{1}} f\left(x_{1}, x_{2}\right) \cdot h_{1}+D_{E_{2}} f\left(x_{1}, x_{2}\right) \cdot h_{2},
$$

for $\left(h_{1}, h_{2}\right) \in E_{1} \times E_{2}$.

Or, with the obvious matrix notation,

$$
D F\left(x_{1}, x_{2}\right)\left(h_{1}, h_{2}\right)=\left[D_{E_{1}} f\left(x_{1}, x_{2}\right) \quad D_{E_{2}} f\left(x_{1}, x_{2}\right)\right]\left[\begin{array}{l}
h_{1} \\
h_{2}
\end{array}\right] .
$$

Exercise 13.3. (The Implicit Function Theorem) Let $X, Y$, and $Z$ be Banach spaces. (You may content yourself with considering $X=\mathbb{R}^{n}, Y=\mathbb{R}^{m}$ and $Z=\mathbb{R}^{m}$.)

Let $U \subseteq X \times Y$ be an open set, and let $f: U \rightarrow Z$ be a differentiable mapping of class $C^{r}, r \geq 1$.

Let $\left(x_{0}, y_{0}\right)$ be a point in $U$, such that $f\left(x_{0}, y_{0}\right)=0$, and assume that the partial differential $D_{Y} f\left(x_{0}, y_{0}\right): Y \rightarrow Z$ of $f$ along $Y$ at $\left(x_{0}, y_{0}\right) \in U$ is a toplinear isomorphism.

Define the mapping $\Phi: U \rightarrow X \times Z$ by $\Phi(x, y)=(x, f(x, y))$. Then $\Phi$ is clearly differentiable of class $C^{r}$, and the differential of $\Phi$ at $\left(x_{0}, y_{0}\right) \in U$ is a continuous linear mapping

$$
D \Phi\left(x_{0}, y_{0}\right): X \times Y \rightarrow X \times Z .
$$


1) Show that the differential of $\Phi$ at $\left(x_{0}, y_{0}\right) \in U$ is given by

$$
D \Phi\left(x_{0}, y_{0}\right) \cdot(h, k)=\left(h, D_{X} f\left(x_{0}, y_{0}\right) \cdot h+D_{Y} f\left(x_{0}, y_{0}\right) \cdot k\right)
$$

for $(h, k) \in X \times Y$.

Or, with the obvious matrix notation,

$$
D \Phi\left(x_{0}, y_{0}\right)(h, k)=\left[\begin{array}{cc}
1_{X} & 0 \\
D_{X} f\left(x_{0}, y_{0}\right) & D_{Y} f\left(x_{0}, y_{0}\right)
\end{array}\right]\left[\begin{array}{l}
h \\
k
\end{array}\right],
$$

where $1_{X}$ denotes the identity mapping of $X$ onto itself.

2) Show that $D \Phi\left(x_{0}, y_{0}\right)$ is a toplinear isomorphism.

Hint: Using matrix notation, the inverse continuous linear mapping can be defined by

$$
\left[\begin{array}{cc}
1_{X} & 0 \\
-\left(D_{Y} f\left(x_{0}, y_{0}\right)\right)^{-1} \circ D_{X} f\left(x_{0}, y_{0}\right) & \left(D_{Y} f\left(x_{0}, y_{0}\right)\right)^{-1}
\end{array}\right] .
$$

3) Show that there exist an open neighbourhood $W \subseteq X$ of $x_{0} \in X$, an open neighbourhood $V \subseteq U \subseteq X \times Y$ of $\left(x_{0}, y_{0}\right) \in U$, and a differentiable mapping $g: W \rightarrow Y$ of class $C^{r}$, such that the following holds:

$$
[(x, y) \in V \quad \text { and } f(x, y)=0] \Longleftrightarrow[x \in W \quad \text { and } y=g(x)] .
$$

Further show that the differential of $g$ at $x \in W$ is given by

$$
D g(x)=-D_{Y} f(x, g(x))^{-1} \circ D_{X} f(x, g(x)) .
$$

\section{Problem Set 14}

Exercise 14.1. Think of $\mathbb{R}^{9}$ as the product $\mathbb{R}^{9}=\mathbb{R}^{3} \times \mathbb{R}^{3} \times \mathbb{R}^{3}$ with coordinates $(x, y, z) \in \mathbb{R}^{9}$, in which $x=\left(x_{1}, x_{2}, x_{3}\right), y=\left(y_{1}, y_{2}, y_{3}\right)$ and $z=$ $\left(z_{1}, z_{2}, z_{3}\right)$ are vectors in $\mathbb{R}^{3}$. Let $\langle\cdot, \cdot\rangle$ denote the standard inner product in $\mathbb{R}^{3}$.

Define the subset $M \subset \mathbb{R}^{9}$ by

$$
M=\left\{\begin{array}{l|l}
(x, y, z) \in \mathbb{R}^{9} & \begin{array}{l}
\langle x, x\rangle=\langle y, y\rangle=\langle z, z\rangle=1 \\
\langle x, y\rangle=\langle x, z\rangle=\langle y, z\rangle=0
\end{array}
\end{array}\right\}
$$


1) Show that $M$ is a compact, 3-dimensional, differentiable manifold of class $C^{\infty}$.

2) A linear mapping $L: \mathbb{R}^{3} \rightarrow \mathbb{R}^{3}$ such that $\langle L(v), L(w)\rangle=\langle v, w\rangle$ for all $v, w \in \mathbb{R}^{3}$ is called a linear isometry in $\mathbb{R}^{3}$. Let $\mathbf{O}(3)$ denote the space of linear isometries in $\mathbb{R}^{3}$ equipped with the induced topology when considered as a subset of $L\left(\mathbb{R}^{3}, \mathbb{R}^{3}\right)$.

Let $\left\{e_{1}, e_{2}, e_{3}\right\}$ denote the canonical basis in $\mathbb{R}^{3}$. Show that the mapping

$$
\Phi: \mathbf{O}(3) \rightarrow M \quad \text { defined by } \Phi(L)=\left(L\left(e_{1}\right), L\left(e_{2}\right), L\left(e_{3}\right)\right)
$$

is a homeomorphism.

3)(Optional) Let $\mathbf{O}(n)$ denote the space of linear isometries in $\mathbb{R}^{n}$ equipped with the canonical inner product. Show that $\mathbf{O}(n)$ can be given the structure of a compact, differentiable manifold of class $C^{\infty}$ and dimension

$$
n^{2}-n-\left(\begin{array}{l}
n \\
2
\end{array}\right)=\frac{n \cdot(n-1)}{2}
$$

With composition of isometries as multiplication, the manifold $\mathbf{O}(n)$ is also a group, and the group operations are differentiable. Such a group manifold is called a Lie group after the Norwegian mathematician Sophus Lie (1842-1899).

The particular Lie group $\mathbf{O}(n)$ is known as the orthogonal group in $n$-dimensional Euclidean space.

Exercise 14.2. Let $M$ be an $n$-dimensional, differentiable manifold of class $C^{r}, r \geq 1$. Show that for every point $x \in M$ there exists an open neighbourhood $U$ of $x \in M$ and $n$ differentiable functions $\varphi_{1}, \ldots, \varphi_{n}: M \rightarrow \mathbb{R}$ of class $C^{r}$, such that the restriction of $\varphi=\left(\varphi_{1}, \ldots, \varphi_{n}\right): M \rightarrow \mathbb{R}^{n}$ to $U \subseteq M$ defines a chart $(U, \varphi)$ on $M$ about $x \in M$.

Hint: You may wish to make use of a localization function $\rho$ of the type defined below.

First define the function $\lambda: \mathbb{R} \rightarrow \mathbb{R}$ by

$$
\lambda(x)= \begin{cases}\exp \left(-1 / x^{2}\right) & \text { for } x>0 \\ 0 & \text { for } x \leq 0\end{cases}
$$

Then it is well known that $\lambda: \mathbb{R} \rightarrow \mathbb{R}$ is differentiable of class $C^{\infty}$.

Now let $a$ and $b$ be arbitrary real numbers with $0<a<b$. Then define the function $\rho: \mathbb{R}^{n} \rightarrow \mathbb{R}$ by

$$
\rho(x)=\frac{\lambda\left(b^{2}-\|x\|^{2}\right)}{\lambda\left(b^{2}-\|x\|^{2}\right)+\lambda\left(\|x\|^{2}-a^{2}\right)},
$$


where $\|\cdot\|$ is the Euclidean norm in $\mathbb{R}^{n}$. Then $\rho: \mathbb{R}^{n} \rightarrow \mathbb{R}$ is differentiable of class $C^{\infty}$, and

$$
\rho(x)= \begin{cases}1 & \text { for }\|x\| \leq a \\ 0 & \text { for }\|x\| \geq b\end{cases}
$$

Exercise 14.3. Let $M$ be a compact, $n$-dimensional, differentiable manifold of class $C^{r}, r \geq 1$.

1) Show that there exist a finite number of open sets $U_{1}, \ldots, U_{k}$ in $M$ that cover $M$, and with associated differentiable functions of class $C^{r}$,

$$
\varphi_{i j}: M \rightarrow \mathbb{R} \quad \text { and } \quad \rho_{i}: M \rightarrow \mathbb{R}, \quad i=1, \ldots, k, j=1, \ldots, n,
$$

such that
a) the restriction of $\varphi_{i}=\left(\varphi_{i 1}, \ldots, \varphi_{i n}\right): M \rightarrow \mathbb{R}^{n}$ to $U_{i}$ defines a chart $\left(U_{i}, \varphi_{i}\right)$ on $M$,
b) $\rho_{i}(x)=0$ for $x \notin U_{i}$,
c) $\sum_{i=1}^{k} \rho_{i}(x)>0$ for all $x \in M$.

2) In continuation of 1 ), define $f: M \rightarrow \mathbb{R}^{(n+1) k}$ by

$$
f(x)=\left(\varphi_{11}(x), \ldots, \varphi_{1 n}(x), \ldots, \varphi_{k 1}(x), \ldots, \varphi_{k n}(x), \rho_{1}(x), \ldots, \rho_{k}(x)\right),
$$

for $x \in M$.

Show that $f$ is injective and a homeomorphism of $M$ onto its image $f(M)$ in $\mathbb{R}^{(n+1) k}$ with the induced topology.

3) (Optional) Show that $f$ is an embedding of class $C^{r}$.

Exercise 14.4. Let $M$ be an $n$-dimensional, differentiable manifold of class $C^{r}, r \geq 1$.

Let $\mathcal{E}(M, p)$ denote the space of germs of differentiable functions $f: \Omega \rightarrow \mathbb{R}$ of class $C^{r}$ defined in open neighbourhoods $\Omega$ of $p \in M$. By a derivation in $\mathcal{E}(M, p)$ we understand a mapping $X_{p}: \mathcal{E}(M, p) \rightarrow \mathbb{R}$ which for all $\lambda, \mu \in \mathbb{R}$ and $f, g \in \mathcal{E}(M, p)$ satisfies:

$$
\begin{aligned}
& X_{p}[\lambda f+\mu g]=\lambda X_{p}[f]+\mu X_{p}[g] \\
& X_{p}[f \cdot g]=X_{p}[f] \cdot g+f \cdot X_{p}[g] .
\end{aligned}
$$

Let $\operatorname{Der}(M, p)$ denote the space of derivations in $\mathcal{E}(M, p)$.

1) Show that $\operatorname{Der}(M, p)$ can be equipped in a natural way with structure as a real vector space. 
2) Show that the mapping $T_{p} M \rightarrow \mathcal{D} \operatorname{er}(M, p)$, which to the tangent vector $v_{p} \in T_{p} M$ associates $v_{p}$ considered as a derivation in $\mathcal{E}(M, p)$, is linear.

3) Now consider the situation in a chart $(U, \varphi)$ on $M$ around $p \in M$ only. Let $\left(x_{1}, \ldots, x_{n}\right) \in \varphi(U) \subseteq \mathbb{R}^{n}$ be the coordinates belonging to the chart, and assume that $\varphi(p)=(0, \ldots, 0)$.

Then show that every germ $f \in \mathcal{E}(M, p)$ can be written in the form $f=$ $\sum_{i=1}^{n} x_{i} g_{i}$ in which $g_{i} \in \mathcal{E}(M, p)$ for $i=1, \ldots, n$, and $x_{i}$ is the germ at $p \in M$ of the function that associates the $i$ th coordinate to every point in $U$.

Further show that

$$
g_{i}(p)=\frac{\partial}{\partial x_{i}}(p)[f] .
$$

4) (Continuation of 3)). Show that an arbitrary derivation $X_{p}$ in $\mathcal{E}(M, p)$ can be written in a unique way as a linear combination

$$
X_{p}=\sum_{i=1}^{n} a_{i} \frac{\partial}{\partial x_{i}}(p), a_{i} \in \mathbb{R}, i=1, \ldots, n,
$$

and conclude from this that the linear mapping

$$
T_{p} M \rightarrow \operatorname{Der}(M, p),
$$

defined in 2), is an isomorphism.

\section{Problem Set 15}

Exercise 15.1. Let $U \subseteq \mathbb{R}^{n}$ be an open set in $\mathbb{R}^{n}$, and let $f: U \rightarrow \mathbb{R}^{n}$ be a differentiable mapping of class $C^{1}$. (One may think of $f$ as a vector field on $U$ by placing the vector $f(x) \in \mathbb{R}^{n}$ at every point $x \in U$.) A zero of $f$, i.e. a point $x_{0} \in U$, such that $f\left(x_{0}\right)=0$, is called non-degenerate if the differential of $f$ at $x_{0}, D f\left(x_{0}\right): \mathbb{R}^{n} \rightarrow \mathbb{R}^{n}$, is an isomorphism.

Assume that possible zeros of $f$ are all non-degenerate. Then show that $f$ has at most finitely many zeros on any compact subset $K \subseteq U$.

Exercise 15.2. Consider $\mathbb{R}^{2}$ with coordinates $(u, x) \in \mathbb{R}^{2}$.

1) Determine the rank of the mapping $F: \mathbb{R}^{2} \rightarrow \mathbb{R}^{2}$ at all points $(u, x) \in \mathbb{R}^{2}$, when $F$ is defined by (i) $F(u, x)=(u, x)$; (ii) $F(u, x)=\left(u, x^{2}\right)$; (iii) $F(u, x)=$ $\left(u, u x-x^{3}\right)$, respectively.

2) In the cases (ii) and (iii) we set

$$
K=\left\{(u, x) \in \mathbb{R}^{2} \mid \mathrm{rk}_{(u, x)} F=1\right\} .
$$


Show that $K$ is a smooth curve in both cases. Find a simple parametrization $\alpha: \mathbb{R} \rightarrow \mathbb{R}^{2}$ of $K$, and determine the rank of $F \circ \alpha$ corresponding to all parameter values.

3) (optional) Show that the germs $\left(\mathbb{R}^{2}, 0\right) \rightarrow\left(\mathbb{R}^{2}, 0\right)$ defined by the above three smooth mappings are not pairwise equivalent.

Hint: Make use, for example, of a geometrical interpretation of the germs.

Exercise 15.3. Show that $f:\left(\mathbb{R}^{2}, 0\right) \rightarrow(\mathbb{R}, 0)$ defined by $f(x, y)=x^{2}-x y$ is a Morse germ.

Determine the index of $f$, and provide a change of coordinates that will bring $f$ into normal form.

\section{Problem Set 16}

Exercise 16.1. Consider two media separated by a plane and suppose that the velocity of light is $c_{1}$ in the first medium and $c_{2}$ in the second. A light ray passes from a point $P$ in the first medium to the point $Q$ in the second medium. Let $i$ be the angle of incidence (the angle of the incoming light ray to the normal of the plane of separation) and $r$ the angle of refraction (the angle of the refracted light ray to the normal of the plane of separation). Deduce the law of refraction from Fermat's principle of light propagation (the Principle of Least Time):

$$
\frac{\sin i}{\sin r}=\frac{c_{1}}{c_{2}}
$$

This law is called Snell's law after the Dutch mathematician Willebrord Snell (1580-1626) who established it in 1621.

Exercise 16.2. Consider a right triangle with the constant perimeter $L$. How shall the triangle be designed, in the class of right triangles, in order to enclose the maximal possible area?

Exercise 16.3. Make a study of the minima for a suitable energy function on the space of maps $C^{2}\left(S^{1}, S^{1} \times[0, h]\right)$ consisting of maps $f: S^{1} \rightarrow S^{1} \times[0, h]$ of class $C^{2}$ of the circle $S^{1}$ into the circular cylinder $S^{1} \times[0, h]$ of height $h>0$. Relate the result obtained to the problem of winding a rubber band around a circular cylinder of height $h>0$. 


\section{Problem Set 17}

Exercise 17.1. Let $[a, b]$ be a closed and bounded interval in $\mathbb{R}$. Denote by $C^{1}\left([a, b], \mathbb{R}^{n}\right)$ the vector space of differentiable curves $x:[a, b] \rightarrow \mathbb{R}^{n}$ in $\mathbb{R}^{n}$ of class $C^{1}$. Equip $C^{1}\left([a, b], \mathbb{R}^{n}\right)$ with the norm

$$
\|x\|_{1}=\sup \left\{\|x(t)\|+\left\|x^{\prime}(t)\right\| \mid t \in[a, b]\right\},
$$

in which $\|\cdot\|$ is the maximum norm in $\mathbb{R}^{n}$.

For an arbitrary open set $U$ in $\mathbb{R} \times \mathbb{R}^{n} \times \mathbb{R}^{n}$, we denote by $\tilde{U}$ the subset of curves $x \in C^{1}\left([a, b], \mathbb{R}^{n}\right)$, in which $\left(t, x(t), x^{\prime}(t)\right) \in U$ for all $t \in[a, b]$.

1) Show that $\tilde{U}$ is an open set in $C^{1}\left([a, b], \mathbb{R}^{n}\right)$.

Now let $U$ be an open set in $\mathbb{R} \times \mathbb{R}^{n} \times \mathbb{R}^{n}$, considered with coordinates $(t, q, p) \in \mathbb{R} \times \mathbb{R}^{n} \times \mathbb{R}^{n}$, and let $L=L(t, q, p): U \rightarrow \mathbb{R}$ be a differentiable function of class $C^{1}$.

Define the function $f: \tilde{U} \rightarrow \mathbb{R}$ by

$$
f(x)=\int_{a}^{b} L\left(t, x(t), x^{\prime}(t)\right) d t \quad \text { for } x \in \tilde{U}
$$

2) Show that $f: \tilde{U} \rightarrow \mathbb{R}$ is differentiable in $\tilde{U}$ with the differential determined by

$$
D f(x) h=\int_{a}^{b} D L\left(t, x(t), x^{\prime}(t)\right) \cdot\left(0, h(t), h^{\prime}(t)\right) d t
$$

for $x \in \tilde{U}$ and $h \in C^{1}\left([a, b], \mathbb{R}^{n}\right)$.

In the following, the curves $x \in \tilde{U}$ and $h \in C^{1}\left([a, b], \mathbb{R}^{n}\right)$ are kept fixed.

3) Show that there exists an $\varepsilon>0$, such that the curve $x+\lambda h$ belongs to $\tilde{U}$, for all $\lambda \in]-\varepsilon, \varepsilon[$.

With reference to 3 ), define the function $g:]-\varepsilon, \varepsilon[\rightarrow \mathbb{R}$ by

$$
g(\lambda)=f(x+\lambda h) \text { for } \lambda \in]-\varepsilon, \varepsilon[.
$$

4) Show that $g$ is differentiable at $\lambda=0$ with the differential quotient

$$
\begin{aligned}
g^{\prime}(0) & =\int_{a}^{b} D L\left(t, x(t), x^{\prime}(t)\right) \cdot\left(0, h(t), h^{\prime}(t)\right) d t \\
& =\int_{a}^{b}\left[\sum_{i=1}^{n}\left(\frac{\partial L}{\partial q_{i}} h_{i}+\frac{\partial L}{\partial p_{i}} h_{i}{ }^{\prime}\right)\right] d t .
\end{aligned}
$$

Here, as well as in 5), the partial derivatives of $L$ shall be taken at the points $\left(t, x(t), x^{\prime}(t)\right) \in U$ and the functions $h_{i}, h_{i}{ }^{\prime}$ at $t \in[a, b]$. 
5) Now assume that $h(a)=h(b)=0$ and that $L=L(t, q, p): U \rightarrow \mathbb{R}$ is a differentiable function of class $C^{2}$.

Using integration by parts, first show that

$$
\int_{a}^{b} \frac{\partial L}{\partial p_{i}} h_{i}^{\prime} d t=-\int_{a}^{b} \frac{d}{d t}\left(\frac{\partial L}{\partial p_{i}}\right) h_{i} d t
$$

and next that

$$
g^{\prime}(0)=\int_{a}^{b}\left[\sum_{i=1}^{n}\left(\frac{\partial L}{\partial q_{i}}-\frac{d}{d t}\left(\frac{\partial L}{\partial p_{i}}\right)\right) h_{i}\right] d t .
$$

The system of equations

$$
\frac{\partial L}{\partial q_{i}}-\frac{d}{d t}\left(\frac{\partial L}{\partial p_{i}}\right)=0 \quad, i=1, \ldots, n,
$$

is called the Euler-Lagrange equations for the above function $f=f(x)$ defined by $L$. It is of fundamental importance in the calculus of variations.

6) Show that the differentiable curve $x:[a, b] \rightarrow \mathbb{R}^{n}$ in $\tilde{U}$ is a stationary point of $f: \tilde{U} \rightarrow \mathbb{R}$, i.e. $D f(x)=0$, if and only if

$$
\frac{\partial L}{\partial q_{i}}\left(t, x(t), x^{\prime}(t)\right)=\frac{d}{d t}\left(\frac{\partial L}{\partial p_{i}}\left(t, x(t), x^{\prime}(t)\right)\right),
$$

for all $i=1, \ldots, n$. 
This page is intentionally left blank 


\section{Bibliography}

In the bibliography below, every book is listed under the subject of special relevance to the present book. The bibliography is by no means exhaustive.

\section{General topology}

J.D. Dugundji: "Topology," Allyn and Bacon, Boston, 1966.

G.F. Simmons: "Introduction to Topology and Modern Analysis," McGrawHill, New York, 1963.

W.A. Sutherland: "Introduction to Metric and Topological Spaces," Oxford University Press, 1975.

\section{Analysis in Banach spaces}

H. Cartan: "Calcul différentiel," Herman, Paris, 1967.

J. Dieudonné: "Foundations of Modern Analysis," Academic Press, New York, 1960.

S. Lang: "Analysis II," Addison-Wesley, Reading Massachusetts, 1969.

\section{Analysis on manifolds, foundations of global analysis}

R. Abraham, J.E. Marsden, T. Ratiu: "Manifolds, Tensor Analysis, and Applications," Addison-Wesley, Reading Massachusetts, 1983.

N. Bourbaki: "Varietés différentielles et analytiques," Herman, Paris, 1967.

Y. Choquet-Bruhat, C. de Witt-Morette, M. Dillard-Bleick: "Analysis, Manifolds and Physics," North-Holland, Amsterdam, 1977. 
J. Dieudonné: "Treatise on Analysis," Vol. III, Academic Press, New York, 1972.

S. Lang: "Introduction to differentiable manifolds," Interscience Publishers, New York-London, 1962.

\section{Differential geometry and topology}

T. Bröcker, K. Jänich: "Introduction to differential topology," Cambridge University Press, 1982.

B.A. Dubrovin, A.T. Fomenko, S.P. Novikov: "Modern Geometry-Methods and Applications," Vol. I, II, Springer, Berlin-Heidelberg-New York, Vol. I 1984, Vol. II 1985.

V. Guillemin, A. Pollack: "Differential Topology," Prentice-Hall, New Jersey, 1974.

M.W. Hirsch: "Differential Topology," Springer, Berlin-Heidelberg-New York, 1976.

J. Milnor: "Topology from the differentiable viewpoint," (Revised reprint of the 1965 original) Princeton University Press, Princeton, NJ, 1997.

F.W. Warner: "Foundations of Differentiable Manifolds and Lie Groups," ScottForesman, Dallas Texas, 1971.

\section{Dynamical systems}

V.I. Arnold: "Geometrical methods in the theory of ordinary differential equations," Springer, Berlin-Heidelberg-New York, 1983.

D.R.J. Chillingworth: "Differential topology with a view to applications," Pitman, London, 1976.

R.L. Devaney: "An introduction to chaotic dynamical systems," (Second edition) Addison-Wesley, Redwood City, CA, 1989.

J. Guckenheimer, P. Holmes: "Nonlinear oscillations, dynamical systems, and bifurcations of vector fields," Springer, Berlin-Heidelberg-New York, 1983.

M.C. Irwin: "Smooth dynamical systems," Academic Press, New York, 1980.

D.J. Luo, L.B. Teng: "Qualitative theory of dynamical systems," World Scientific, Singapore, 1993. 
J. Palis, W. de Melo: "Geometric theory of dynamical systems, an introduction," Springer, Berlin-Heidelberg-New York, 1982.

D. Ruelle: "Elements of differentiable dynamics and bifurcation theory," Academic Press, Inc., Boston, MA, 1989.

\section{Singularity theory and catastrophe theory}

V.I. Arnold: "Catastrophe Theory," Springer, Berlin-Heidelberg-New York, 1984.

V.I. Arnold, A.N. Varchenko, S.M. Gusein-Zade: "Singularities of Differentiable Maps," Vol. I, II, Birkhäuser Boston Inc., Cambridge Massachusetts, Vol. I 1985, Vol. II 1988.

M. Golubitsky, V. Guillemin: "Stable Mappings and Their Singularities," Springer, Heidelberg-New York, 1973.

M. Golubitsky, D.G. Schaeffer: "Singularities and Groups in Bifurcation Theory," Vol. I, Springer, Berlin-Heidelberg-New York, 1985.

V.L. Hansen: "Geometry in Nature," AK Peters Ltd., Wellesley, Massachusetts, 1993.

M. Golubitsky, I. Stewart, D.G. Schaeffer: "Singularities and Groups in Bifurcation Theory," Vol. II, Springer, Berlin-Heidelberg-New York, 1988.

T. Poston, I. Stewart: "Catastrophe Theory and its Applications," Dover Publications, Inc., Mineola, New York, 1996 (reprint of the 1978 original).

\section{Calculus of variations}

J. Eells, L. Lemaire: "Selected topics in harmonic maps," CBMS Regional Conference Series in Mathematics, No. 50, American Mathematical Society, Providence, Rhode Island, 1983.

V.L. Hansen: "Shadows of the Circle - Conic Sections, Optimal Figures and Non-Euclidean Geometry," World Scientific, Singapore, 1998.

W. Klingenberg: "Lectures on closed geodesics," Springer, Berlin-HeidelbergNew York, 1978.

J.W. Milnor: "Morse Theory," Princeton University Press, 1963.

R.S. Palais: "Foundations of global non-linear analysis," Benjamin, New York, 1968. 
H. Seifert, W. Threlfall: "Variationsrechnung im Grossen," Teubner, Leipzig, 1938 .

J.L. Troutman: "Variational calculations with elementary convexity," Springer, Berlin-Heidelberg-New York, 1983.

L.C. Yong: "Lectures on the Calculus of Variations and Optimal Control Theory," Saunders, Philadelphia, 1969.

\section{Theoretical mechanics}

R. Abraham, J. Marsden: "Foundations of Mechanics," (Second edition) Benjamin/Cummings, Reading Massachusetts, 1978.

V.I. Arnold: "Mathematical methods of classical mechanics," Springer, BerlinHeidelberg-New York, 1978. 


\section{Index}

accumulation point, 25

Aleksandrov, P.S., 32

algebra, 134

algebraic variety, 118

Anti-commutativity, 142

approaching

convergence, 28,29

atlas, 117

for differentiable structure, 124

on manifold, 123

axioms of countability, 21

Baire set, 180

Baire space, 180

Baire, R.-L., 180

ball

$$
\begin{aligned}
& \text { open, } 12 \\
& \text { unit, } 63
\end{aligned}
$$

Banach Fixed Point Theorem, 88

Banach space, 90

Banach, S., 85

basis of neighbourhoods, local basis, 21

basis for topology, 21

Bernoulli, Jakob, 194

Bernoulli, Johann, 194

bijective, 44

Bolzano, B., 32

Borel, E., 32

boundary

of subset, 23

point, 23

bounded

linear mapping, 63

set, 37,202

Brouwer, L., 197

$C^{1}$-topology, 160

$C^{2}$-topology, 198

Cauchy condition

for sequences, 87

for series, 104

Cauchy sequence, 86

Cauchy, A.-L., 86

Cauchy-Schwarz inequality, 9

centre of ball, 12

centred charts, 151

Chain Rule, 74, 83, 144

chart, 117,123

Clifford torus, 119

closed, 23

closure of subset, 26

coarsest topology, 16

codimension, 153

compact, 37

sequentially, 36

topological space, 41

comparison test, 104

compatible atlases, 124

complete metric space, 86

composite mapping

continuity, 19

differentiability, 74

connected, 47

pathwise, 48

constraints, 121 
contact point, 25

continuity, 6, 12, 14, 18, 24

at a point, $6,12,20$

uniform, 6, 43

contraction, 88

factor, 88

converge, 29

convergence

of functions, 29

of sequences, 29

of series, 104

convex, 100

coordinate, 56

coordinate function vector field, 140

coordinate system local, 123

coordinate vector fields, 135

countable, 21, 34

covering

open, 36

critical point, 55, 184

non-degenerate, 184

degree of circle map, 197

dense, 27, 180

derivation, 134, 141, 221

derivative, 52,70

$2^{\text {nd }}, 82$

$3^{\text {rd }}, 82$

$r$ th, 82

first, 76

higher order, 82

partial, 71

diffeomorphic manifolds, 145

diffeomorphism, 55, 108, 145

local, 109

differentiability, 67

$r$ times, 82

at a point, 53

class $C^{r}, 82$

class $C^{1}, 76$ class $C^{\infty}, 82$

differentiable

manifold, 117, 125

mapping of class $C^{r}, 128$

vector field, 139

differentiable structure, 124

differential, 54, 68, 76, 143, 146

differential equations

existence and uniqueness

of solutions, 216

Dini's theorem, 207

Dini, U., 207

direct method in

calculus of variations, 195, 208

directional derivative, 133,140

discrete

metric, 11

topology, 16

distance, 8

between subsets, 209

to a subset, 205

Eells, J., 128

embedding, 150

energy function, 54, 196

$\varepsilon$-function, 53

$\varepsilon$-mapping, 67

equivalence classes, 3

equivalence of germs, 165

equivalence relation, 2

equivalent

norms, 59

topological spaces, 44

Euclidean

metric, 10

norm, 58

space, 10

topology, 16

Euler, L., 194

Euler-Lagrange equations, 225

Euler-Poincaré characteristic, 161

evaluation mapping, 202 
exterior

$$
\text { of subset, } 22
$$

point, 22

extremum

local, 121

Fermat's principle, 188

Fermat, P. de, 187

finest topology, 16

finite order

$$
\text { germ of, } 168
$$

first axiom of countability, 21

first derivative, 76

fixed point, 88

foliation, 149

Fréchet, M., 8

fundamental sequence, 86

fundamental system of neighbourhoods, 21

Fundamental Theorem of Calculus, 100

general linear group, 103

generic, 27, 157, 181

geodesic, 54

germ, 133,164 singular, 166

gradient, 71, 119

greatest lower bound, 4,10

Hausdorff space, 30

Hausdorff, F., 30

Heine, E., 32

Hesse matrix, 171

Hessian, 171

Hilbert manifold, 55

Hilbert space, 55, 92

Hilbert, D., 92

homeomorphic topological spaces, 44

homeomorphism, 44

Hopf, H., 32 identity

isomorphism, 103

mapping, 44

image, 2

immersed submanifold, 151

immersion, 147, 183

Implicit Function Theorem, 218

index of Morse germ, 172

indiscrete topology, 16

induced

differentiable structure, 126

mapping, 143, 146

metric, 12

topology, 16

infimum, 4,10

injective, 44

inner product, 9

integral, 96

integral operator, 213

interior

$$
\text { of subset, } 21
$$

point, 21

interval trap, 33

inverse

mapping, 44

transformation, 103

Inverse Function Theorem, 109

for germs, 165

isolated point, 25

isometry, 220

isoperimetric problem, 189

iterate, 88

Jacobi's identity, 142

Jacobian, 73

Jacobian matrix, 73

jet-prolongation, 182

Lagrange multipliers, 121

Lagrange, J.-L., 122

largest topology, 16

least upper bound, 3,10 
Lebesque, H., 32

Leibnitz, G.W., 194

Lie

algebra, 142

group, 220

product, 142

Lie, S., 220

limit point, 28,29

Lindelöf's covering theorem, 208

Lindelöf, E., 208

Lipschitz continuous, 216

local

basis of neighbourhoods, 21

coordinate system, 123

diffeomorphism, 109

extremum, 121

localization function, 220

locally trivial

vector bundle, 138

lower semicontinuous

function, 207

majorant, 3

manifold

n-dimensional, 123

differentiable, 117

of maps, 54, 128, 198

sub-, 118, 151

topological, 117, 122

maximal atlas, 124

maximal element, 3

maximum

local, 121,213

norm, 58

Mean Value Theorem, 98

metric, 8

discrete, 11

Euclidean, 10

metric space, 8

in the extended sense, 177

metrizable

topological space, 16 minimal element, 3

minimal surface, 119

minimum

local, 121, 213

minorant, 4

Morse

function, 184

germ, 171

theory, 55, 195

Morse Lemma, 173

Morse, M., 55

multilinear mapping, 78

multipliers

Lagrange, 121

neighbourhood, 19 open, 19

Newton, I., 194

non-degenerate

zero of vector field, 222

critical point, 184

norm, 9,57

Euclidean, 58

maximum, 58

operator, 65,78

sum, 58

norm of partition, 93

normal form

of germ, 166

normalizable

topological vector space, 132

normed vector space, 57

numerable, 21

open

ball, 12

covering, 36

neighbourhood, 19

set, 13, 15

sphere, 12

Open Mapping Theorem, 102

operator norm, 65,78 
order of germ, 168

ordered set, 3

ordering relation, 3

orthogonal group, 220

overlap of class $C^{r}, 124$

parameter curves, 134

parameterized curve, 69

partial

differential, 218

derivative, 71

sum, 104

partition, 93 norm of, 93

pathwise connected, 48

point

accumulation, 25

boundary, 23

contact, 25

critical, 55

exterior, 22

fixed, 88

interior, 21

isolated, 25

limit, 28,29

pointwise defined, 59

positive definite, 8,57

precompact, 208

preimage, 2

Principle of Least Time, 188

product structure, 127

product topology, 17

quotient topology, 18

radius of ball, 12

rank of differentiable

mapping, 116, 147

regular germ, 166

regular point, 164

regular value, 116

relation, 2

residual, 180
Riemann

$$
\text { integral, } 96
$$

sum, 93

Riemann, B., 93

right equivalence of germs, 165

second axiom of countability, 21

second derivative, 82

Separation Axiom $\mathrm{T}_{1}, 32$

sequence

Cauchy, 86

fundamental, 86

sequentially compact, 36

series, 104

set

compact, 37

connected, 47

open, 13,15

zero, 116

shift operator, 63

singular germ, 166

singular point, 164, 184

smallest topology, 16

smooth manifold, 125

Snell's law, 223

Snell, W., 223

space

Banach, 90

compact topological, 41

complete metric, 86

connected, 47

Euclidean, 10

Hilbert, 55, 92

normed vector, 57

$\mathrm{T}_{1}, 32$

$\mathrm{T}_{2}, 32$

topological, 15

sphere

open, 12

unit, 60

stability, 157

stable, 28 
Steiner, J., 192

subcovering, 36

submanifold, 118, 151

immersed, 151

submersion, 148

subspace topology, 16

sum

norm, 58

Riemann, 93

sum of series, 104

supremum, 3,10

surface

minimal, 119

surjective, 44

symmetry of metric, 8

$\mathrm{T}_{1}$-space, 32

$\mathrm{T}_{2}$-space, 32

tangent, 52, 70

tangent bundle, 135

base space, 135

projection, 135

total space, 135

tangent space, 120, 131

tangent vector, 130

foot of, 131

tangential curves, 129

tangential mapping, 143, 146

term in series, 104

third derivative, 82

Thom's Transversality Theorem, 157

Thom, R., 157

toplinear isomorphism, 102, 132

topological

manifold, 117, 122

space, 15

vector space, 132

topology, 5, 12, 14, 15

discrete, 16

Euclidean, 16

indiscrete, 16

induced, 16 largest, 16

product, 17

quotient, 18

smallest, 16

subspace, 16

trace, 16

torus, 119,153

Clifford, 119

trace topology, 16

transversal, 153

transversal intersection, 157

transversality, 153, 157

triangle inequality, 8,57

uniform

$C^{k}$-topology, 177

continuity, 6,43

scaling, 57

unit ball, 63

unit sphere, 60

variety

algebraic, 118

vector bundle, 138

bundle map, 146

fibre, 138

locally trivial, 138

section, 139

vector field, 139

local representative, 139

coordinate function, 140

vector space

topological, 132

Weierstrass, K., 32

Whitney

$C^{k}$-topology, 178

$C^{\infty}$-topology, 178

Whitney Immersion Theorem, 184

Whitney, H., 116

zero-section, 160

zero-set, 116 